\title{
Tsallis nonextensive statistical mechanics of El Niño Southern Oscillation Index
}

\author{
M. Ausloos and F. Petroni* \\ SUPRATECS, B5, Sart Tilman, \\ B-4000 Liège, Euroland
}

\begin{abstract}
The shape and tails of partial distribution functions (PDF) for a climatological signal, i.e. the El Niño SOI and the turbulent nature of the ocean-atmosphere variability are linked through a model encompassing Tsallis nonextensive statistics and leading to evolution equations of the Langevin and Fokker-Planck type. A model originally proposed to describe the intermittent behavior of turbulent flows describes the behavior of the normalized variability for such a climatological index, for small and large time windows, both for small and large variability. This normalized variability distributions can be sufficiently well fitted with a $\chi^{2}$-distribution. The transition between the small time scale model of nonextensive, intermittent process and the large scale Gaussian extensive homogeneous fluctuation picture is found to occur at above ca. a 48 months time lag. The intermittency exponent $(\kappa)$ in the framework of the Kolmogorov log-normal model is found to be related to the scaling exponent of the PDF moments. The value of $\kappa(=0.25)$ is in agreement with the intermittency exponent recently obtained for other atmospheric data.
\end{abstract}

PACS numbers: PACS :05.45.Tp, 05.10.Gg, 89.65.Gh

*Also at Dipartimento di Matematica, Universitá dell'Aquila, 67010 L'Aquila, Italy 


\section{INTRODUCTION}

Fractional Gaussian noises and fractional Brownian motions [1] have served recently as models for a wide variety of data in various fields, like meteorology [2, 3], geology [4], cardiac dynamics [5], finance [6-8]. The concept of fractional Gaussian noise (fGn) as formulated by Mandelbrot and Van Ness [1] is a derivative process obtained from fractional Brownian motion $(\mathrm{fBm}) B_{H}(t)$, namely $\lim _{\delta \longrightarrow 1}\left(B_{H}(t+\delta)-B_{H}(t)\right) / \delta$. As it has been shown by Flandrin [9]. Even though the fBm is a non-stationary process it obeys a power law over all frequencies. In this study we use fGn as a model for a climatological signal.

The origin of non-Gaussian, thus non negligible large and sometimes so called extreme volatility events characterized by so called fat tailed distributions is a key question in statistical physics; the fat tails of (short and long-range) volatilities are thought to be caused by some 'dynamical process'. Destroying all correlations, e.g. by shuffling the order of the fluctuations, is known to cause the fat tails almost to vanish.

The fat tails indicate an unexpected high probability of large changes. These extreme events are of utmost importance for risk analysis. They are considered to be a set of strong bursts in the energy dissipation. In so doing the PDF and the fat tail event existence are thought to be similar to the notion of intermittency in turbulent flows [10].

It is an open question whether both the fat-tailed power-law of partial distribution functions (PDF) of the various volatilities and their evolution for different time delays in climatological indices can be described.

On the other hand, the non-Gaussian character of the fully developed turbulence [11] has been linked to nonextensive statistical physics [12-20]. It seems that there is no study of Tsallis statistics application or approach in climatology [21].

One of the most intriguing phenomena in climatology, known as El Niño, i.e. the more or less cyclic warming and cooling of the eastern and central regions in the Pacific Ocean, appears to be very complicated to describe [22-25]. There are three sorts of models, based on special types of filters designed specifically to detect a signal from given atmospheric input. The ability of these models for producing successful forecasts of El Niño appears to be concomitant to the very low-frequency and large-scale evolution of the characteristic patterns in the atmospheric boundary layer circulation. This evolution can be thought of as a signal that precedes $\mathrm{El} \mathrm{Niño} \mathrm{events.} \mathrm{It} \mathrm{would} \mathrm{be} \mathrm{useful} \mathrm{to} \mathrm{have} \mathrm{model-independent} \mathrm{results}$ 
with basic geophysical inputs.

El Niño is a disruption of the ocean-atmospheric system in the tropical Pacific having important consequences for weather around the globe. It is factually described by the so called Southern oscillation index (SOI). Much of the drastic and tragic events occurring in North America, Tropical Africa and Europe are also attributed to El Niño. There are different ways of characterizing El Niño events. One of them is by the values of an index such as the Southern Oscillation Index (a proxy measure of El Niño based on surface air pressure differences between Darwin, Australia and Tahiti, French Polynesia) [29], or large sea surface temperatures (SST) and sea surface height (SSH) anomalies in the eastern tropical Pacific Ocean. Here we will use the sea level pressure (SLP) differences between two meteorological stations, one at Tahiti, the other at Darwin. Questions on variability of the SOI on various time scales are relevant for better modelling. It was recently found for the southern oscillation index (SOI), characterizing El Niño events [26] that long-range correlations exist between the fluctuations of the index. Also, correlation between the SOI and the North Atlantic Oscillation (NAO) index has been reported [27] having a common oscillation of about $6-8$ years. The NAO was studied per se, in [28].

In this paper the behavior of a climatological index, i.e. the SOI, on short and large time windows (time scales to be better defined below) is studied along the lines of a recently suggested model of hydrodynamic turbulence that serves as a dynamic foundation for nonextensive statistics [16-18].

In Sect. 2, we describe the distribution of variability for the monthly value signal of the SOI index for the time interval between Jan. 1866 and Jan. 2006, thus a series of $N=1681$ months or data points, values downloaded from the Climate Prediction Center web site (http : //www.cpc.ncep.noaa.gov/data/indices/). We also characterize the tail(s) of the distribution for various time lags $\Delta t$ 's, i.e. from 1 month up to 36 months (or 3 years!), and will observe the value of the PDF tails, for such time lags, outside the best gaussian fit through the data. There are several ways of displaying features in the variability of a climatological index. A simple one represents value increment $\Delta y(t)=y(t+\Delta t)-y(t)$ or difference between the value of the index $y(t)$ at time $t+\Delta t$ and its value at time $t$. Below we mainly consider the normalized variability $Z(t, \Delta t)=\left(\Delta y(t)-<\Delta y>_{\Delta t}\right) / \sigma_{\Delta t}$, where $<\Delta y>_{\Delta t}$ denotes the average and $\sigma_{\Delta t}$ the standard deviation of $\Delta y(t)$ for a given $\Delta t$. The normalized variability $Z(t, \Delta t)$ depend on the time $t$ and the time lag $\Delta t$. However, 
in order to simplify the notations and whenever possible without leading to confusion and misunderstanding we will drop the explicit writing of one or both variables.

In Sect. 3, we calculate the power law exponents characterizing the integrated distribution of the normalized variability over different time lags for the SOI index monthly values through a detrended fluctuation analysis and a power spectral density analysis point of view.

In all cases it is useful to test the null hypothesis or estimate the error bars with respect to standard signals. It is thought [10] that the fat tails are caused by long-range volatility correlations. Destroying all correlations by shuffling the order of the fluctuations, is known to cause the fat tails almost to vanish. A Kolmogorov-Smirnov test (not shown) on shuffled data has indicated us the statistical validity of the numerical values and the statistically acceptable meaning of the displayed error bars. Results are compared to shuffled data for estimating the value of the error bars.

In Sect. 4, Tsallis statistical approach is outlined, and distributions of (normalized) variability for time lags between $\Delta t=1$ to 36 months are examined. The nonextensivity, i.e. some anomalous scaling of classically extensive properties like the entropy, is linked to a single parameter $q$, e.g. in the Tsallis formulation of nonextensive thermostatistics. It is found that the $q$-value of the nonextensive entropy converges to a value $=1.01$ for $\Delta t$ $=36$ months, starting with $q=1.25$ for $\Delta t=1$. The probability density $f_{\Delta t}(\beta)$ of the volatility $\beta$ in terms of the standard deviation of the normalized variability of the SOI for different time lags is found to obey the $\chi^{2}$-distribution. The intermittency exponent $(\kappa)$ of the Kolmogorov log-normal model is found to be related to the scaling exponent of the PDF moments, -thereby giving weight to this model.

In Sect. 5, the usual Fokker-Planck approach for treating the time-dependent probability distribution functions is summarized. Coefficients governing both the Fokker-Planck equation for the distribution function of normalized variability and the Langevin equation for the time evolution of normalized variability of monthly value signal of SOI are obtained. Therefore we present for the first time a coherent theory linking the shape and tails of partial distribution functions for long and short time lags of the monthly values of a climatological signal and connect the often suggested turbulent nature of the ocean-atmospheric interface interactions to a model encompassing nonextensive statistics and evolution equations of the Langevin and Fokker-Planck type.

We will often compare results based on normalized variability and non normalized vari- 
ability time series.

\section{DATA AND DISTRIBUTION OF VARIABILITY}

Monthly values of the SOI index for the time interval between Jan. 1866 and Jan. 2006 were downloaded from the Joint Institute for Study of the Atmosphere and Ocean (JISAO) web site http : //tao.atmos.washington.edu/pacs/additional_analyses/soi.html for the longest period available there, i.e. from Jan. 1866 to June 1999. Data for the time interval from July 1999 to Jan. 2006 were downloaded from the Climate Prediction Center NCEP web site (http://www.cpc.ncep.noaa.gov/data/indices/). For the years before 1866 daily measurements of the sea level pressure at both stations have been reported to exist and monthly values of the southern oscillation index have been calculated [34] back to 1841. However, there are gaps of a couple of years in the record whence not suitable for our analysis. We have chosen the JISAO's data for the period before July 1999, since they do not contain missing values as the data series from NCEP do. The data are fully compatible because the standardization of both JISAO and NCEP data series is calculated through the standard deviation $S$ of the sea level pressure (SLP) at a station in Tahiti and the sea level pressure at a station in Darwin

$$
S O I=\frac{P_{\text {Tahiti }}-P_{\text {Darwin }}}{S}
$$

Such SOI monthly data are plotted as a function of time in Fig. 1. The data set consists of 1681 data points. It is sometimes stated that daily or weekly values of the SOI do not convey much in the way of useful information about the current state of the climate, and accordingly the Bureau of Meteorology does not issue them. Daily values in particular can fluctuate markedly because of daily weather patterns, and should not be used for climate purposes. We may disagree with this statement (provided the reliability of the data). There are indeed techniques which can sort out noise from coherent behavior [30].

Sustained negative values of the SOI often indicate El Niño episodes [31]. These negative values are usually accompanied by sustained warming of the central and eastern tropical Pacific Ocean, a decrease in the strength of the Pacific Trade Winds, and a reduction in rainfall over eastern and northern Australia. The most recent strong El Niño was in 1997/98. Positive values of the SOI are associated with stronger Pacific trade winds and warmer sea 
temperatures to the north of Australia, popularly known as a La Niña episode. Waters in the central and eastern tropical Pacific Ocean become cooler during this time. Together these give an increased probability that eastern and northern Australia will be wetter than normal. The most recent strong La Niña was in 1988/89; a moderate La Niña event occurred in 1998/99, which weakened back to neutral conditions before reforming for a shorter period in 1999/2000. This last event finished in Autumn 2000.

The distribution of the normalized variability $Z(t, \Delta t)$ of the monthly value signal of SOI index between Jan. 1866 and Jan. 2006, for $\Delta t=1$ month are plotted in Fig. 2a. The partial distribution of non normalized variability $\Delta y$ of the monthly value signal of SOI index is plotted in Fig $2 \mathrm{~b}$ as we will later compare some of our findings for both $Z$ and $\Delta y$. For comparison A fit is first attempted with a Gaussian distribution for small values of the increments, i.e. the central part of the distribution. The distribution is well fitted with such a Gaussian type curve within the interval $Z \in]-2,2[$ but departs from the Gaussian form outside this interval. The negative and positive tails of the distribution outside the Gaussian curve are found both to be equal to -5.6 . In the case $\Delta t \geq 1$ month, it is observed that the best Gaussian range is increasing with increasing time lag (Fig. 3).

\section{TIME CORRELATIONS AND SPECTRAL POWER}

There are different estimators for the long and/or short range dependence of fluctuations correlations [36].

Through the (linearly) detrended fluctuation analysis (DFA) method, see e.g. [37], we show first that the long range correlations of monthly value signal of SOI for the time interval of interest, are $1 / f$-like. The method has been used previously to identify whether long range correlations exist in non-stationary signals, in many research fields such as e.g.

finance [7, 8], cardiac dynamics [5] and of course meteorology [2, 3, 38]. Its concepts are therefore not repeated here. For an extensive list of references see [37]. Briefly, the signal time series $y(t)$ is first integrated, to 'mimic' a random walk $Y(t)$. The time axis (form 1 to $N$ ) is next divided into non-overlapping boxes of equal size $n$; one looks thereafter for the best (linear) trend, $z_{n}$, in each box, and calculates the root mean square deviation of the (integrated) signal with respect to $z_{n}$ in each box. The average of such values is taken at fixed box size $n$ in order to obtain 


$$
F(n)=\sqrt{\frac{1}{N} \sum_{i=1}^{N}\left[Y(i)-z_{n}(i)\right]^{2}}
$$

The box size is next varied over the $n$ value. The resulting function is expected to behave like $n^{1+H_{D F A}}$ indicating a scaling law. For the (integrated) monthly value signal of the SOI index, a scaling exponent $1+H_{D F A}=1.05 \pm 0.01$ is found (Fig. 4) in a scaling range extending from about 4 to about 66-72 months. A signal with Hausdorff dimension $H_{D F A}$ close to zero has the characteristics of a fractional Gaussian noise signal $[4,39]$.

Along the same line of thought the scaling properties of the normalized variability $Z(t, \Delta t)=\left(\Delta y(t)-<\Delta y>_{\Delta t}\right) / \sigma_{\Delta t}$ have also been tested for different time lag values, i.e. $\Delta t=1,3,6,12,24,36$ months (Fig. 5). The DFA functions, as defined here above, of the integrated normalized variability shows non trivial scaling properties for the series of normalized variability. The values of the scaling exponents and the maximum box size $n_{x}$ (in days) for which the scaling holds for each DFA-function are given in Table I, while the DFA-functions together with fitting lines are plotted in Fig. 5.

The power spectrum of the monthly value signal of SOI $S(f) \sim f^{-\mu}$ with spectral exponents $\mu_{1}=-0.26$ and $\mu_{2}=1.20$ with a scale break at $1 / 70$ months $^{-1}$ is shown in Fig. 6 . The scaling properties of the power spectrum of two surrogate data, one in which the amplitudes are randomly shuffled and another in which the magnitudes are preserved but the sign of the data is shuffled, are shown in the inserts of Fig. 6. Such scaling spectral exponents $\mu=0$ are signature of a white noise like behavior. Recall that $\mu=2.0$ corresponds to usual Brownian motion. The theoretical relationship $\mu=2 H_{D F A}-1$ is approximately verified, the weak agreement being likely due to the quite limited data size.

We have also checked for scaling behavior and possible periodicities in the power spectrum of the time series of the normalized variability $Z(t, \Delta t)=\left(\Delta y(t)-<\Delta y>_{\Delta t}\right) / \sigma_{\Delta t}$ for different (selected) values of the time lag $\Delta t=1,3,6,12,24,36$ months (Fig. 7).

Periodicities in the power spectrum of the normalized variability time series for $\Delta t>$ 1 month were expected to be found since these periods are somewhat embedded into the time series by the way they are obtained and the Fourier transform technique. It is easily observed that the maxima and the minima of the spectrum correspond to harmonics and subharmonics of $1 / \Delta t$. 


\section{TSALLIS STATISTICS}

Based on the scaling properties of multifractals [40] Tsallis [12, 41] proposed a generalized Boltzmann-Gibbs thermo-statistics through the introduction of a family of non-extensive entropy functional $\mathcal{S}_{q}$ given by:

$$
\mathcal{S}_{q}=k \frac{1}{q-1}\left(1-\int p(x, t)^{q} d x\right)
$$

with a single parameter $q$ and where $k$ is a normalization constant. The main ingredient in Eq.(3) is the time-dependent probability distribution $p(x, t)$ of the stochastic variable $x$. The functional is reduced to the classical extensive Boltzmann-Gibbs form in the limit of $q \longrightarrow 1$. The Tsallis parameter $q$ characterizes the non-extensivity of the entropy. Subject to certain constraints the functional in Eq.(3) seems to yield a probability distribution function of the form $[10,12,16,32,33]$

$$
p(x)=\frac{1}{Z_{q}}\left\{1+\frac{C \beta_{0} 2 \alpha(q-1)|x|^{2 \alpha}}{2 \alpha-(q-1)}\right\}^{-\frac{1}{(q-1)}}
$$

for the stochastic variable $x$, where

$$
\frac{1}{Z_{q}}=\alpha\left\{\frac{C \beta_{0} 2 \alpha(q-1)}{2 \alpha-(q-1)}\right\}^{1 / 2 \alpha} \frac{\Gamma\left(\frac{1}{q-1}\right)}{\Gamma\left(\frac{1}{2 \alpha}\right) \Gamma\left(\frac{1}{q-1}-\frac{1}{2 \alpha}\right)}
$$

in which $C$ is a constant and $0<\alpha \leq 1$ is the power law exponent of the potential $U(x)=$ $C|x|^{2 \alpha}$ that provides the 'restoring force' $F(x)$ in Beck model of turbulence [16-18, 20]. The latter is described by a Langevin equation

$$
\frac{d x}{d t}=-\gamma F(x)+R(t)
$$

where $\gamma$ is a parameter and $R(t)$ is a gaussian white noise. A non-zero value of $\gamma$ corresponds to providing energy to (or draining from) the system by the outside [42]. The parameter $\beta_{0}$ in Eq.(4) and (5) is the mean of the fluctuating standard deviation $\beta$, i.e. the local standard deviation of $|x|$ over a certain window of size $m$ [10]. We will use this model assuming that the normalized variability $Z(t, \Delta t)$ represent the stochastic variable $x$, as in Eq.(1). We will search whether Eq.(4) is obeyed for $x \equiv Z(t, \Delta t)$, thus studying $p(x) \equiv p_{\Delta t}(Z)$ for various time lags $\Delta t$. 
Just as in Beck model of turbulence [16-18] we assume that the standard deviation $\beta$ is $\chi^{2}$-distributed with degree $\nu$ (see another formula in [20]):

$$
f_{\Delta t}(\beta) \equiv \frac{1}{\Gamma(\nu / 2)}\left(\frac{\nu}{2 \beta_{0}}\right)^{\nu / 2} \beta^{\nu / 2-1} \exp \left(-\frac{\nu \beta}{2 \beta_{0}}\right), \quad \nu>2,
$$

where $\Gamma$ is the Gamma function, $\beta_{0}=<\beta>$ and the number of degrees of freedom $\nu$ can be found from:

$$
\nu=\frac{2<\beta>^{2}}{<\beta^{2}>-<\beta>^{2}} .
$$

The Tsallis parameter $q$ satisfies [16]

$$
q \equiv 1+\frac{2 \alpha}{\alpha \nu+1} .
$$

To justify our assumption that the 'local' standard deviation of the normalized variability $Z(t, \Delta t)$ is of the form of $\chi^{2}$-distribution, we checked the distribution of the normalized variability of the monthly value signal of SOI. We have calculated the standard deviation of the normalized variability within various non-overlapping windows of size $m$, ranging from 6 to 36 months

$$
\beta(k)=\sqrt{\frac{1}{m} \sum_{i=k m+1}^{(k+1) m} Z^{2}(i)-\left(\frac{1}{m} \sum_{i=k m+1}^{(k+1) m} Z(i)\right)^{2}}
$$

In doing so we have a various number of $\mathcal{M}$ non-overlapping windows for various time lags $\Delta t$, and have searched for the most efficient size of the window in order not to loose data points and therefore, information. The resulting empirically obtained distributions of the 'local' standard deviation (Eq.(10)) of normalized variability for the different time lags of interest are plotted in Fig. 8 for an intermediary case $m=12$. The values of the degree $\nu$ of the $\chi^{2}$-distribution are then obtained using Eq. (8). The spread $\left[\beta_{\min }, \beta_{\max }\right]$ of the local standard deviation $\beta$ decreases with increasing the time lag as it is expected from a $\chi^{2}$-distribution function due to the exponential function in Eq. (7) for large values of the degree of freedom $\nu$. The value of $\nu$ much varies as a function of $m$ and the time lags considered. The fits are always very good. However the $\beta_{0}$ and $\nu$ values are quite dependent on the parameters used in the numerical analysis. Based on these results, e.g. Fig. 8, it can be accepted that the (turbulent) model $\beta$-distributions can be sufficiently well fitted for our purpose with a $\chi^{2}$-distribution, thereby justifying the initial assumption.[57] 
The probability distributions of the normalized variability for the different values of the time lag $\Delta t=1,3,6,12,24,36$ months are shown in Fig. 3 together with the lines representing the best fit to the Tsallis type of distribution function. In Table II the statistical parameters related to the Tsallis type of distribution function are summarized, including a criterion for the goodness of the fit, i.e. the Kolmogorov-Smirnov distance $d_{K S}$, which is defined as the maximum distance between the cumulative probability distributions of the data and the fitting lines. Note that the kurtosis (see Table II) for the Tsallis type of distribution function

$$
K_{r}=K_{L} \frac{(5-3 q)}{(7-5 q)},
$$

where $K_{L}=3$ for a Gaussian process, is positive for all values of $q<7 / 5$ as expected, since its positiveness is directly related to the occurrence of intermittency [10]. Moreover, the limit $q<7 / 5$ also implies that the second moment of the Tsallis type distribution function will always remain finite, as necessarily due to the type of phenomena hereby studied.

Furthermore, if we assume that the Kolmogorov log-normal model of turbulence [47] is applicable and let $\Delta t_{L}$ be the scale at which the whole partial distribution function becomes Gaussian, then the kurtosis $K_{r}$ should scale as

$$
K_{r}=K_{L}\left(\frac{\Delta t}{\Delta t_{L}}\right)^{-\delta} .
$$

Therefore

$$
q=\frac{5-7\left(\Delta t / \Delta t_{L}\right)^{-\delta}}{3-5\left(\Delta t / \Delta t_{L}\right)^{-\delta}} .
$$

In order to obtain an estimate for $\Delta t_{L}$, we increase the time lag to the value $\Delta t=$ 48 months, quite outside the range so far examined (see Fig.3 for example) leading to a rather complete coincidence between the distribution functions in the Tsallis and Gaussian forms for the presently investigated data. The corresponding parameter values are listed in Table II. A quick perusal observation convincingly indicates where the transition occurs between the small time scale model of nonextensive, intermittent process and the large scale Gaussian extensive homogeneous fluctuation picture [10, 12].

In Fig. 9 the Tsallis parameter $q$ is shown as a function of the rescaled time lags $\Delta t / \Delta t_{L}$, where $\Delta t_{L}$ is the integral scale, the scale at which the whole probability distribution function converges to Gaussian. The crosses represent the $q$ values for which the best fit to the SOI 
data (Fig. 3) is obtained with Eq. (4). With this the value of the integral scale $\Delta t_{L}$, we find the value of the exponent $\delta=0.11$ as the one for which the Eq. (13) fits best the $q$-values. The exponent value $\delta=0.11$ also allows to fit well the power law dependence (Eqs. (11) and (12)) of the rescaled kurtosis $K_{r} / K_{L}$ as shown in the insert of Fig. 9.

Note that in the framework of the Kolmogorov log-normal model [17, 47], $\delta=4 \kappa / 9$, where $\kappa$ is called the intermittency exponent. Therefore, we find $\kappa=0.25$ for the intermittency exponent of normalized variability of the SOI signal in the time interval of interest. This value of $\kappa$ is interestingly the same as the value of the intermittency exponent $\kappa=0.25$ for turbulence recently obtained from experimental atmospheric data [48]. Early estimates have varied from 0.18 to 0.85 using different experimental techniques [49-51]. Large range of values of the intermittency exponent, ranging from 0.2 to 0.8 , have been reported in studies of multiparticle production [52]. It was found that the range of intermittency exponent values depend on the number of cascades; the smaller the number of stages of the multiplicative cascade the smaller $\kappa$, and conversely [Fig. 2b in [52]].

One can explore the Tsallis type of the probability distribution function Eq.(4) in two limits. For small values of normalized $\log$ variability $Z$ the probability distribution function converges to the form

$$
p_{\Delta t}(Z) \approx \frac{1}{Z_{q}} \exp \left\{-\frac{C \beta_{0} 2 \alpha}{2 \alpha-(q-1)}|Z|^{2 \alpha}\right\}
$$

Therefore the Tsallis type distribution function converges to a Gaussian, i.e. $\alpha \longrightarrow 1$, for small values of the normalized log variability, for any $\Delta t$ investigated hereby (see Figs. 2-3).

In the limit of large values of normalized variability $Z$, the Tsallis type distribution converges to a power law

$$
p_{\Delta t}(Z) \approx \frac{1}{Z_{q}}\left\{\frac{(q-1) C \beta_{0} 2 \alpha}{2 \alpha-(q-1)}|Z|^{2 \alpha}\right\}^{-\frac{1}{q-1}} .
$$

Studying the Tsallis type of distribution function one can obtain from Eq.(4) an expression for the width of the Tsallis type of probability distribution function, $2 \sigma_{w}^{2}=$ $(2 \alpha-(q-1)) /\left(2 \alpha C \beta_{0}(q-1)\right)$. In the limit of $\alpha \longrightarrow 1$ the width of the Tsallis type distribution $2 \sigma_{w}^{2}=(3-q) / 2 C \beta_{0}(q-1)$, i.e. $\sim 2 /\left(C \beta_{0}\right)$. It is obvious that for large time lags $2 \sigma_{w}^{2}$ tends to diverge [32], like $\simeq(\Delta t)^{2 /(3-q)}$; this can be easily verified on a log-log plot (not shown). 
In limit of $q \longrightarrow 1$ the Tsallis type distribution function converges to Gaussian. The values of the parameters $q, \alpha, C \beta_{0}$, that best fit the data using Eq.(4), and $2 \sigma_{w}^{2}$ are plotted as a function of the time lag in Fig. 10.

\section{FOKKER-PLANCK APPROACH}

On the other hand, the evolution of a time dependent probability distribution function is usually described within the Fokker-Planck approach. This method provides some further information on the correlations present in the time series and it begins with the joint PDF's, that depend on $\mathcal{N}$ variables, i.e. $p^{\mathcal{N}}\left(Z_{1}, \Delta t_{1} ; \ldots ; Z_{\mathcal{N}}, \Delta t_{\mathcal{N}}\right)$. We started to address this issue by determining the joint $\mathrm{PDF}$ for $\mathcal{N}=2$, i.e. $p\left(Z_{2}, \Delta t_{2} ; \Delta x_{1}, \Delta t_{1}\right)$. The symmetrically tilted character of the joint PDF contour levels (Fig. 11) around an inertia axis with slope +1 points out to some statistical dependence, i.e. a correlation, between the normalized variability $Z(t, \Delta t)$ of the monthly value signal of SOI.

The conditional probability function is

$$
p\left(Z_{i+1}, \Delta t_{i+1} \mid Z_{i}, \Delta t_{i}\right)=\frac{p\left(Z_{i+1}, \Delta t_{i+1} ; Z_{i}, \Delta t_{i}\right)}{p\left(Z_{i}, \Delta t_{i}\right)}
$$

for $i=1, \ldots, \mathcal{N}-1$. For any $\Delta t_{2}<\Delta t_{i}<\Delta t_{1}$, the Chapman-Kolmogorov equation is a necessary condition of a Markov process, one without memory but governed by probabilistic conditions

$$
p\left(Z_{2}, \Delta t_{2} \mid Z_{1}, \Delta t_{1}\right)=\int d\left(Z_{i}\right) p\left(Z_{2}, \Delta t_{2} \mid Z_{i}, \Delta t_{i}\right) p\left(\Delta x_{i}, \Delta t_{i} \mid Z_{1}, \Delta t_{1}\right)
$$

The Chapman-Kolmogorov equation when formulated in dif ferential form yields a master equation, which can take the form of a Fokker-P1anck equation [43]. Let $\tau=\log _{2}(48 / \Delta t)$,

$$
\frac{d}{d \tau} p(Z, \tau)=\left[-\frac{\partial}{\partial Z} D^{(1)}(Z, \tau)+\frac{\partial^{2}}{\partial Z^{2}} D^{(2)}(Z, \tau)\right] p(Z, \tau)
$$

in terms of a drift $D^{(1)}(Z, \tau)$ and a diffusion coefficient $D^{(2)}(Z, \tau)$ (thus values of $\tau$ represent $\left.\Delta t_{i}, i=1, \ldots\right)$.

The coefficient functional dependence can be estimated directly from the moments $M^{(k)}$ (known as Kramers-Moyal coefficients) of the conditional probability distributions: 


$$
\begin{gathered}
M^{(k)}=\frac{1}{\Delta \tau} \int d Z^{\prime}\left(Z^{\prime}-Z\right)^{k} p\left(Z^{\prime}, \tau+\Delta \tau \mid Z, \tau\right) \\
D^{(k)}(Z, \tau)=\frac{1}{k !} \lim M^{(k)}
\end{gathered}
$$

for $\Delta \tau \rightarrow 0$. The drift coefficient $D^{(1)}$ and the diffusion coefficient $D^{(2)}$ are well represented (Fig. 12a,b) by a line and parabola, respectively

$$
\begin{gathered}
D^{(1)}=-0.37 Z-0.01 \\
D^{(2)}=0.10 Z^{2}-0.10 Z+0.33
\end{gathered}
$$

for the normalized variability (plotted with dots).

We have compared the above values of the drift and the diffusion coefficients for those of the drift and the diffusion coefficients for non normalized variability (plotted with open circles) and have obtained

$$
\begin{gathered}
D^{(1)}=-0.52 \Delta y-0.02 \\
D^{(2)}=0.20 \Delta y^{2}-0.10 \Delta y+0.24
\end{gathered}
$$

Note that the first term on the right hand side of Eq. (18) is identified [54] as the term generating drift behavior in the evolution of the PDF, while the second term is responsible for the diffusion, or fluctuation term in the PDF evolution. In the asymptotic case when the linear term in $D^{(1)}$, i.e. the coefficient $D_{1}^{(1)}$ is dominating the dependence and the independent $D_{0}^{(2)}$ term is somewhat dominating in $D^{(2)}$, then the Fokker-Planck equation is linear, otherwise the drift and diffusion terms are intervened. Comparing the values of the linear and independent terms in Eqs. (21) and (23), as well as the values of the quadratic, linear and independent terms in Eqs. (22) and (24) one may argue that the linear approximation for the Fokker-Planck equation holds more convincing for the PDF evolution of the normalized variability (Eqs. $(21,23)$ ) as opposite to the PDF evolution of the non normalized variability $\Delta y$. 
On the other hand, it may be worthwhile to recall that the observed quadratic dependence of the diffusion term $D^{(2)}$ is essential for the logarithmic scaling of the intermittency parameter in studies on turbulence.

Finally, the Fokker-Planck equation for the distribution function is known to be equivalent to a Langevin equation for the variable, i.e. $Z$ here, (within the Ito interpretation $[43-46,53]$ )

$$
\frac{d}{d \tau} Z(\tau)=D^{(1)}(Z(\tau), \tau)+\eta(\tau) \sqrt{D^{(2)}(Z(\tau), \tau)}
$$

where $\eta(\tau)$ is a fluctuating $\delta$-correlated force with Gaussian statistics, i.e. $\left\langle\eta(\tau) \eta\left(\tau^{\prime}\right)>=\right.$ $2 \delta\left(\tau-\tau^{\prime}\right)$.

Thus the Fokker-Planck approach provides the evolution process of PDF's from small time lags to larger ones. The fact that the drift coefficient is finite implies that there is some 'restoring force', i.e. $\gamma \neq 0$ in Eq. (6), while the quadratic dependence of $D^{(2)}$ in $Z$ is obviously like an autocorrelation function for a diffusion process.

An interaction that can produce such a 'restoring force' is the the air-sea interaction that takes place in many different ways. However, its main ingredients are the air-sea fluxes of mass, heat and momentum. Reliable estimates of the air-sea fluxes of heat and momentum are vital to improve our understanding of the coupled ocean-atmosphere system. An air-sea heat and momentum climatology, as the one recently reported [55] can be hopefully used in future dynamic models to relate the findings of this study to classical meteorological qualities using observations.

\section{CONCLUSION}

In summary, we have presented a method that provides the evolution process of probability distribution functions (over 140 years) for one climatological index, i.e. the SOI. It can be first recalled that crossovers in the DFA results and power spectral density point to specific time scales, - in fact related to famous phenomena, like sunspots.

We have mainly studied the evolution process of the tails that are outside the central (Gaussian) regime,- which of course occurs only at small variability, thereby facilitating the understanding of the evolution of these distribution functions in a Fokker-Planck framework. The Gaussian regime range has been found to imply that signal correlations extend up to ca. 48 months, - an interesting time lag to be considered in microscopic evolution model(s) 
of El Niño.

It has been found that Beck turbulence model can be well applied to describe the distributions of the standard deviation of the SOI signal normalized variability assuming a $\chi^{2}$-distribution for these. In some sense this application (or generalization of the model) is justified by the fact that the ideas behind the turbulence model, based on temperature fluctuations, can be expected to be carried over to the case in which pressure fluctuations occur in the system.

An open question in nonextensive thermostatistics studies is often raised about the meaning, value and behavior of the non extensive exponent, or Tsallis parameter $q$. The intermittency exponent is interestingly found to be related to the scaling exponent of the PDF moments in the framework of Kolmogorov log-normal model, thereby giving weight to the model and the statistical approach.

We have also presented the turbulence-like dynamics through the Fokker-Planck and the Langevin equations. We have (as it has been expected) found that, in the treated case, there is some 'restoring force', i.e. ( $\gamma \neq 0$ in the Langevin equation). A comparison is made between normalized variability and non normalized variability.

Whence we have related a climatological signal behavior to Tsallis non extensive thermodynamics approach, i.e. more precisely to a turbulence-like process, - as climatological ocean-atmospheric interface interactions and indices were often claimed to be seen. No need to say that this empirical modeling only describes the evolution of the signal but does not explain it, as a general circulation model [56] should do. Nevertheless the time scales which are hereby observed might shine some light on approximation validity or the need to restrict extrapolations to realistic ranges, - including memory effects. Finally, it seems that we have thoroughly answered the often raised question "why to look at the tails of a probability distribution function? and what does that lead to?'.

\section{Acknowledgements}

Part of FP work has been supported by European Commission Project E2C2 FP6-2003NEST-Path-012975 Extreme Events: Causes and Consequences. Part of this work results from financing through the ARC 02-07/293 Project of the ULg and the COST P10 "Physics 
of Risk" program which MA also thoroughly acknowledges. Critical and encouraging comments by M. Ghil, H. Herrmann, K. Ivanova, J. Peinke, C. Tsallis, and J. Vannimenus have been as always very valuable for improving this report.

[1] B.B. Mandelbrot and J.W. Van Ness, SIAM Rev., 10, 422 (1968)

[2] K. Ivanova, M. Ausloos, E.E. Clothiaux and T.P. Ackerman, Europhys. Lett. 52, 40 (2000)

[3] E. Koscielny-Bunde, A. Bunde, S. Havlin, H. E. Roman, Y. Goldreich and H.-J. Schellnhuber, Phys. Rev. Lett. 81, 729 (1998)

[4] D.L. Turcotte, Fractals and Chaos in Geology and Geophysics (Cambridge University Press, Cambridge, 1997)

[5] P. Ch. Ivanov, M. G. Rosenblum, C.-K. Peng, J. E. Mietus, S. Havlin, H. E.Stanley and A. L. Goldberger, Nature 383, 323 (1996)

[6] K. Ivanova and M. Ausloos, Eur. Phys. J. B 27, 239 (2002)

[7] N. Vandewalle and M. Ausloos, Physica A 246, 454 (1997)

[8] N. Vandewalle and M. Ausloos, Phy. Rev. E 58, 6832 (1998)

[9] P. Flandrin, IEEE Trans. Inform. Theory 35, 197 (1989)

[10] F. M. Ramos, C. Rodrigues Neto, R. R. Rosa, L. D. Abreu Sa and M. J. A. Bolzan,Nonlinear Analysis - Theory 23, 3521 (2001)

[11] U. Frisch, Turbulence: the Legacy of A. N. Kolmogorov, (Cambridge Univ. Press, Cambridge, 1995)

[12] C. Tsallis, J. Stat. Phys. 52, 479 (1988)

[13] C. Tsallis and D.J. Bukman, Phys. Rev. E 54, 2197 (1996)

[14] G. Wilk and Z. Wlodarczyk, Phys. Rev. Lett. 84, 2770 (2000)

[15] T. Arimitsu and N. Arimitsu, Prog. Theor. Phys. 105, 355 (2001)

[16] C. Beck, Phys. Rev. Lett. 87, 180601 (2001)

[17] C. Beck, Physica A 295, 195 (2001)

[18] C. Beck, G. S. Lewis, and H. L. Swinney, Phys. Rev. E 63, 035303 (2001) 
[19] F. Sattin, Physica Scripta 71, 443 (2005)

[20] F. Sattin, Phys. Rev. E 68, 032102 (2003)

[21] A report on the matter was presented by the authors at the 2006 EGU General Assembly, Vienna, April 06.

[22] G.K. Vallis, J. Geophys. Res. 9313979 (1988).

[23] S.G. Philander, Rep. Prog. Phys. 62, 123 (1999).

[24] M. Ghil and N. Jiang Geophys. Res. Lett. 25, 171 (1998).

[25] C.L. Keppenne and M. Ghil J. Geophys. Res. 97, 20449 (1992).

[26] M. Ausloos and K. Ivanova , Phys. Rev. E 63, 047201 (2001).

[27] R. García, P. Ribera, L. Gimenoo, E. Hernández Ann. Geophysicae 18, 247 ( 2000).

[28] C. Collette and M. Ausloos, Int. J. Mod. Phys. C 15, 1353 (2005)

[29] G.T. Walker, Q. J. R. Meteorol. Soc. 54, 79 (1928).

[30] P.J. Brockwell and R.A. Davis, Time Series : Theory and Methods (Springer-Verlag, Berlin, 1991)

[31] http://www.bom.gov.au/climate/glossary/soi.shtml

[32] F. Michael and M.D. Johnson, Physica A 320, 525 (2003).

[33] N Kozuki and N Fuchikami, Physica A 329, 222 (2003).

[34] G.P. Konnen, P.D. Jones, M.H. Kaltofen and R.J. Allan, J. Climate, 11, 2325 (1998)

[35] C. Tsallis, C. Anteneodo, L. Borland and R. Osorio, Physica A 324, 89 (2003).

[36] M.S. Taqqu, V. Teverovsky and W. Willinger, Fractals 3, 785 (1995)

[37] K. Hu, Z. Chen, P. Ch. Ivanov, P. Carpena and H.E. Stanley, Phys. Rev E 64, 011114 (2001)

[38] K. Ivanova and M. Ausloos, Physica A 274, 349 (1999)

[39] G.M. Raymond, D.B. Percival and J.B. Bassingthwaighte, Physica A 322, 169 (2003)

[40] T.C. Halsey, M.H. Jensen, L.P. Kadanoff, I. Procaccia and B.I. Shraiman, Phys. Rev. A 33, 1141 (1986)

[41] C. Tsallis, S.V.F. Levy, A.M.C. Souza and R. Maynard, Phys. Rev. Lett. 75, 3589 (1995)

[42] F. Sattin, J. Phys. A 36, 1583 (2003)

[43] M.H. Ernst, L.K. Haines and J.R. Dorfman, Rev. Mod. Phys. 41, 296 (1969)

[44] L.E. Reichl, A Modern Course in Statistical Physics, Univ. Texas Press, Austin (1980)

[45] P. Hänggi and H. Thomas, Phys. Rep. 88, 207 (1982)

[46] C.W. Gardiner, Handbook of Stochastic Methods, Springer-Verlag, Berlin (1983) 
[47] A.M. Kolmogorov, J. Fluid Mech. 13, 82 (1962)

[48] K.R. Sreenivasan and P. Kailasnath, Phys. Fluids 5, 512 (1993)

[49] K.R. Sreenivasan, R.A. Antonia and H.Q. Danh, Phys. Fluids 20, 1238 (1977)

[50] R.R. Prasad, C. Meneveau and K.R. Sreenivasan, Phys. Rev. Lett 61, 74 (1988)

[51] J.C. Wyngaard and H. Tennekes, Phys. Fluids 13, 273 (1970)

[52] R.A. Janik and B. Ziaja, Acta Phys. Pol. B 30, 259 (1999)

[53] H. Risken, The Fokker-Planck Equation: Methods of Solution and Applications, 2nd edn, (Springer-Verlag, Berlin, 1989)

[54] H.J. Carmichael, Statistical Methods in Quantum Optics 1. Master Equations and FokkerPlanck Equations, (Springer-Verlag, Berlin, 1999).

[55] S.A. Josey, E.C. Kent and P.K.Taylor, Southampton Oceanography Centre Report, 6, 30 (1999)

[56] http://www - das.uwyo.edu/geerts/cwx/notes/chap12/nwp gcm.html

[57] Sattin formula [20] might also be tested in future work. 


\section{Figure Captions}

Figure 1 - Monthly values of the Southern Oscillation Index (SOI) as defined in the text reported from Jan. 1866 to Jan. 2006. Data are downloaded from http : //tao.atmos.washington.edu/pacs/additionalanalyses/soi.html and from ftp : //ftpprd.ncep.noaa.gov/pub/cpc/wd52dg/data/indices/soi after June 1999. Data series consists of 1681 data points

Figure 2 - (a) Probability distribution function of normalized variability $Z(t, \Delta t)$ of monthly values signal of the Southern Oscillation Index from Jan. 1866 to Jan. 2006 for $\Delta t=1$ month (symbols). $Z(t, \Delta t)$ is defined as $Z(t, \Delta t)=\left(\Delta y(t)-<\Delta y>_{\Delta t}\right) / \sigma_{\Delta t}$, where $\Delta y(t)=y(t+\Delta t)-y(t)$ and $\sigma_{\Delta t}$ is the standard deviation of $\Delta y(t)$ for time lag $\Delta t$. The dashed line represents a Gaussian distribution. Inset: Power law fit (solid line) of the negative and positive slope (-5.6 for both) of the distribution outside the Gaussian regime, i.e. $]-2,+2[$. (b) same as (a) but for non normalized variability $\Delta y$

Figure 3 - Probability distribution function $(\mathrm{PDF}) p_{\Delta t}(Z)$ of normalized variability of monthly values signal of the SOI (symbols) and the Tsallis type distribution function (lines) for different values of $\Delta t=1,3,6,12,24,36$ months. The PDF (symbols and curves) for each $\Delta t$ are moved down by 10 with respect to the previous one; the curve for $\Delta t=1$ month is unmoved. The large dots mark the ends of the interval in which the distribution is like a gaussian distribution. The values of the parameters for the Tsallis type distribution function for each $\Delta t$ are summarized in Table II

Figure 4 - DFA function $F(n)$ plotted as a function of the the box size $n$ of the integrated monthly values signal of the SOI from Jan. 1866 to Jan. 2006. $1 / f$-like fluctuations with slope $=1.06 \pm 0.01$ are obtained for time scales below 66 months and fractional Gaussian noise like fluctuations slope $=0.36 \pm 0.02$ above 72 months. Insets: White noise like fluctuations of two types of surrogate data, when the data are shuffled randomly and when the sign of the data is shuffled randomly

Figure 5 - DFA function $F(n)$ plotted as a function of the box size $n$ of the integrated normalized variability $Z(t, \Delta t)$ of the monthly values signal of the SOI from Jan. 1866 
to Jan. 2006, for different time lags $\Delta t=1,3,6,12,24,36$ months. Values of the scaling exponents $H_{D F A}$ for the various DFA functions are summarized in Table I

Figure 6 - Power spectrum $S(f)$ of the monthly values signal of the SOI from Jan. 1866 to Jan. 2006. A scale break at around $f=1 / 70$ month $^{-1}$ separates two scaling regions. Insets: Scaling of the power spectrum of both shuffled amplitude and shuffled sign of monthly values signal of the SOI as a white noise signal with $\mu \approx 0$

Figure 7 - Power spectrum $S(f)$ of the normalized variability $Z(t, \Delta t)$ of the monthly values signal of the SOI from Jan. 1866 to Apr. 2003 for different time lags $\Delta t=$ $1,3,6,12,24,36$ months. Each curve is moved down by $10^{-5}$ with respect to the previous one; the power spectrum of the normalized returns for $\Delta t=1$ month is not displaced

Figure 8 - Probability density $f_{\Delta t}(\beta)$ of the local volatility $\beta$ (Eq.(10)) in terms of standard deviation of the normalized variability $Z(t, \Delta t)$ of SOI in non-overlapping windows with size $m=12$ months for different time lags (symbols) (a-f) $\Delta t=1,3,6,12,24,36$ months. Lines: $\chi^{2}$-distribution as given by Eq. (7)

Figure 9 - The functional dependence of the Tsallis $q$ parameter on the rescaled time lag $\Delta t / \Delta t_{L}$ for $\Delta t_{L}=48$ months and $\delta=0.11$ (see Eq. (13)) (line); the symbols represent the values of the $q$ parameter listed in Table II and used to plot the fitting lines in Fig. 2. Inset : Scaling properties of the rescaled kurtosis $K_{r} / K_{L}$, where $K_{L}=3$ is the kurtosis for a Gaussian process, as a function of the rescaled time lag $\Delta t / \Delta t_{L}$ satisfying Eq. (11) (open symbols) and Eq. (12) (full symbols)

Figure 10 - Characteristic parameters of Tsallis type distribution function as defined in [33] : Tsallis $q$-parameter (crosses), $\alpha$ (squares), constant $C \beta_{0}$ used in the fit (open circles), the width of the Tsallis type distribution $2 \sigma_{w}^{2}=(2 \alpha-(q-1)) /\left(2 \alpha C \beta_{0}(q-1)\right)$ from Eq.(4) (triangles) (rescaled by a factor of $1 / 180$, for better display)

Figure 11 - Typical contour plots of the joint probability density function $p\left(Z_{2}, \Delta t_{2} ; Z_{1}, \Delta t_{1}\right)$ of the monthly values signal of the SOI from Jan. 1866 to Jan. 2006. Dashed lines have a slope +1 and emphasize the correlations between probability density functions for $\Delta t_{2}=1$ month and $\Delta t_{1}=2$ months. Contour levels correspond to 
$\log _{10} p\left(Z_{2}, \Delta t_{2} ; Z_{1}, \Delta t_{1}\right)=-1.8,-2.0,-2.2,-2.4,-2.6,-2.8$ from center to border

Figure 12 - Kramers-Moyal drift (a) $D^{(1)}$ and diffusion (b) $D^{(2)}$ coefficients as a function of normalized variability $Z$ (dots) and non normalized variability $Z$ (open circles) of the monthly values signal of the SOI; $D^{(1)}=-0.37 Z-0.01$ ((a) dots), $D^{(2)}=0.10 Z^{2}-0.10 Z+$ 0.33 ((b) dots); $D^{(1)}=-0.52 \Delta y-0.02\left(\left(\right.\right.$ a) open circles), $D^{(2)}=0.20 \Delta y^{2}-0.10 \Delta y+0.24$ ((b) open circles) 
TABLE I: Values of the scaling exponent from the DFA analysis of normalized variability $Z$ for different values of the time lag $\Delta t=1,3,6,12,24,36$ months, and crossover 'box size' $n_{x}$

$$
\begin{array}{rccc}
\hline \Delta t & 1+H_{D F A 1} & 1+H_{D F A 2} & n_{x} \\
\hline 1 & 0.264 \pm 0.017 & 0.058 \pm 0.016 & 23 \\
3 & 0.594 \pm 0.038 & 0.082 \pm 0.060 & 23 \\
6 & 0.909 \pm 0.028 & 0.101 \pm 0.063 & 23 \\
12 & 1.148 \pm 0.032 & 0.129 \pm 0.070 & 25 \\
24 & 1.115 \pm 0.030 & 0.142 \pm 0.066 & 32 \\
36 & 1.082 \pm 0.027 & 0.192 \pm 0.044 & 35
\end{array}
$$

TABLE II: Values of the parameters characterizing the monthly values of the Southern Oscillation Index (SOI) from Jan. 1866 to Jan. 2006 in the nonextensive thermostatistics approach. For the definition of the Kolmogorov-Smirnov distance $d_{K S}$ see the text

\begin{tabular}{rcccccc}
\hline$\Delta t$ & $q$ & $\alpha$ & $C \beta_{0}$ & $p_{\Delta t}(Z=0)$ & $K_{r}$ & $d_{K S}$ \\
& \multicolumn{7}{c}{ Eq. $(4)$} \\
\hline 1 & 1.25 & 0.93 & 0.70 & 0.448 & 5 & 0.005 \\
3 & 1.20 & 0.91 & 0.65 & 0.432 & 4.20 & 0.012 \\
6 & 1.16 & 0.90 & 0.63 & 0.426 & 3.80 & 0.009 \\
12 & 1.10 & 0.88 & 0.60 & 0.415 & 3.40 & 0.008 \\
24 & 1.06 & 0.87 & 0.58 & 0.407 & 3.21 & 0.009 \\
36 & 1.01 & 0.87 & 0.56 & 0.402 & 3.03 & 0.010 \\
\hline
\end{tabular}




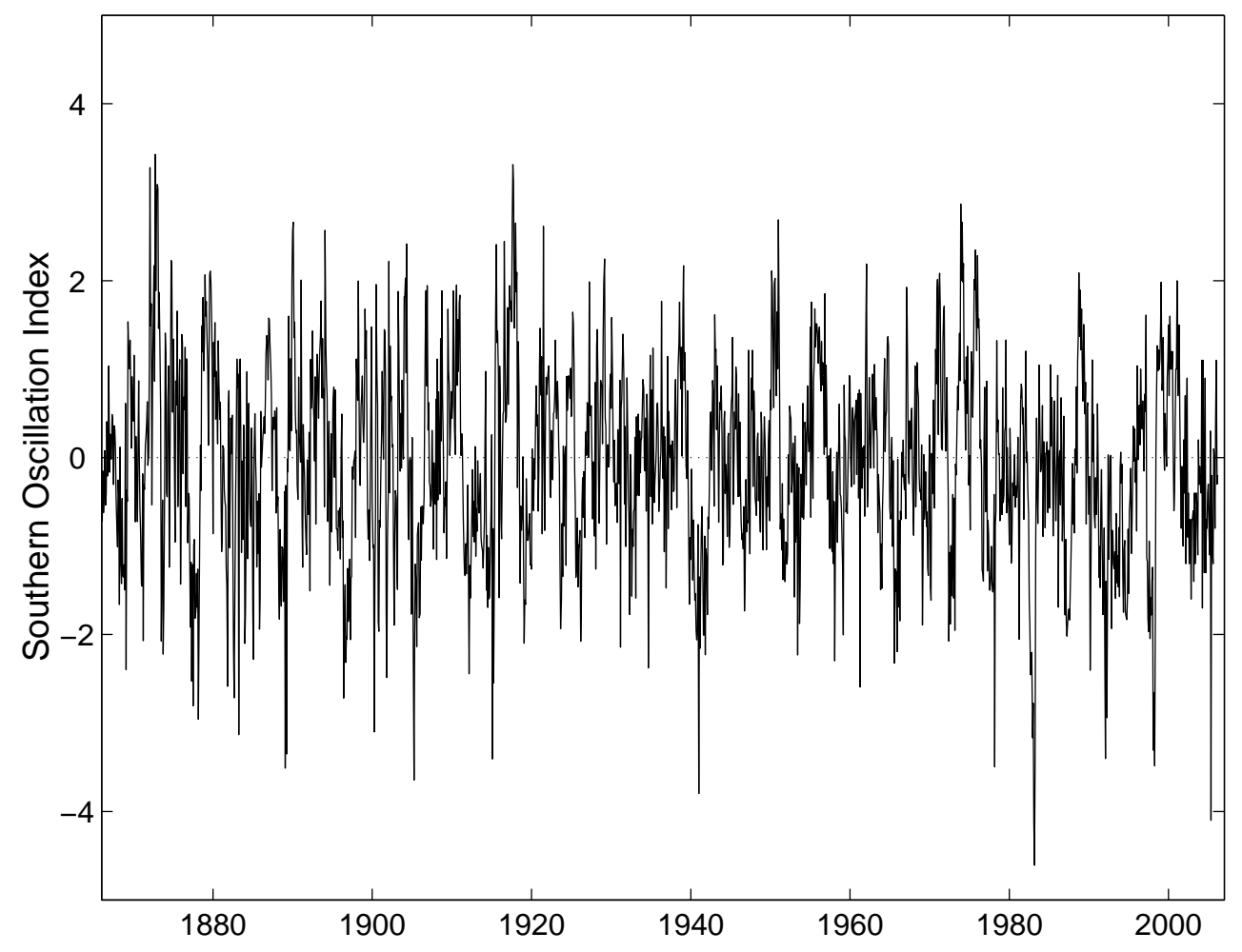

FIG. 1: Monthly values of the Southern Oscillation Index (SOI) as defined in the text reported from Jan. 1866 to Jan. 2006. Data are downloaded from http : //tao.atmos.washington.edu/pacs/additionalanalyses/soi.html and from ftp : //ftpprd.ncep.noaa.gov/pub/cpc/wd52dg/data/indices/soi after June 1999. Data series consists of 1681 data points 

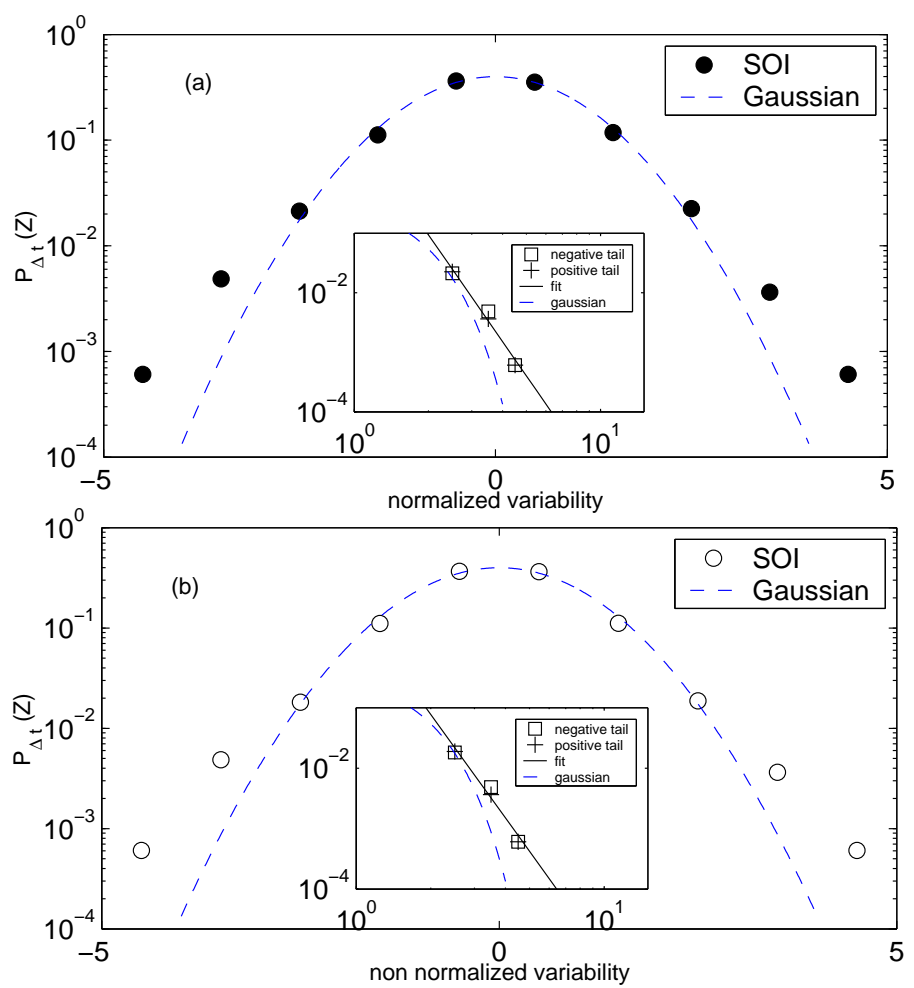

FIG. 2: (a) Probability distribution function of normalized variability $Z(t, \Delta t)$ of monthly values signal of the Southern Oscillation Index from Jan. 1866 to Jan. 2006 for $\Delta t=1$ month (symbols). $Z(t, \Delta t)$ is defined as $Z(t, \Delta t)=\left(\Delta y(t)-<\Delta y>_{\Delta t}\right) / \sigma_{\Delta t}$, where $\Delta y(t)=y(t+\Delta t)-y(t)$ and $\sigma_{\Delta t}$ is the standard deviation of $\Delta y(t)$ for time lag $\Delta t$. The dashed line represents a Gaussian distribution. Inset: Power law fit (solid line) of the negative and positive slope (-5.6 for both) of the distribution outside the Gaussian regime, i.e. ] $-2,+2[$. (b) same as (a) but for non normalized variability $\Delta y$ 


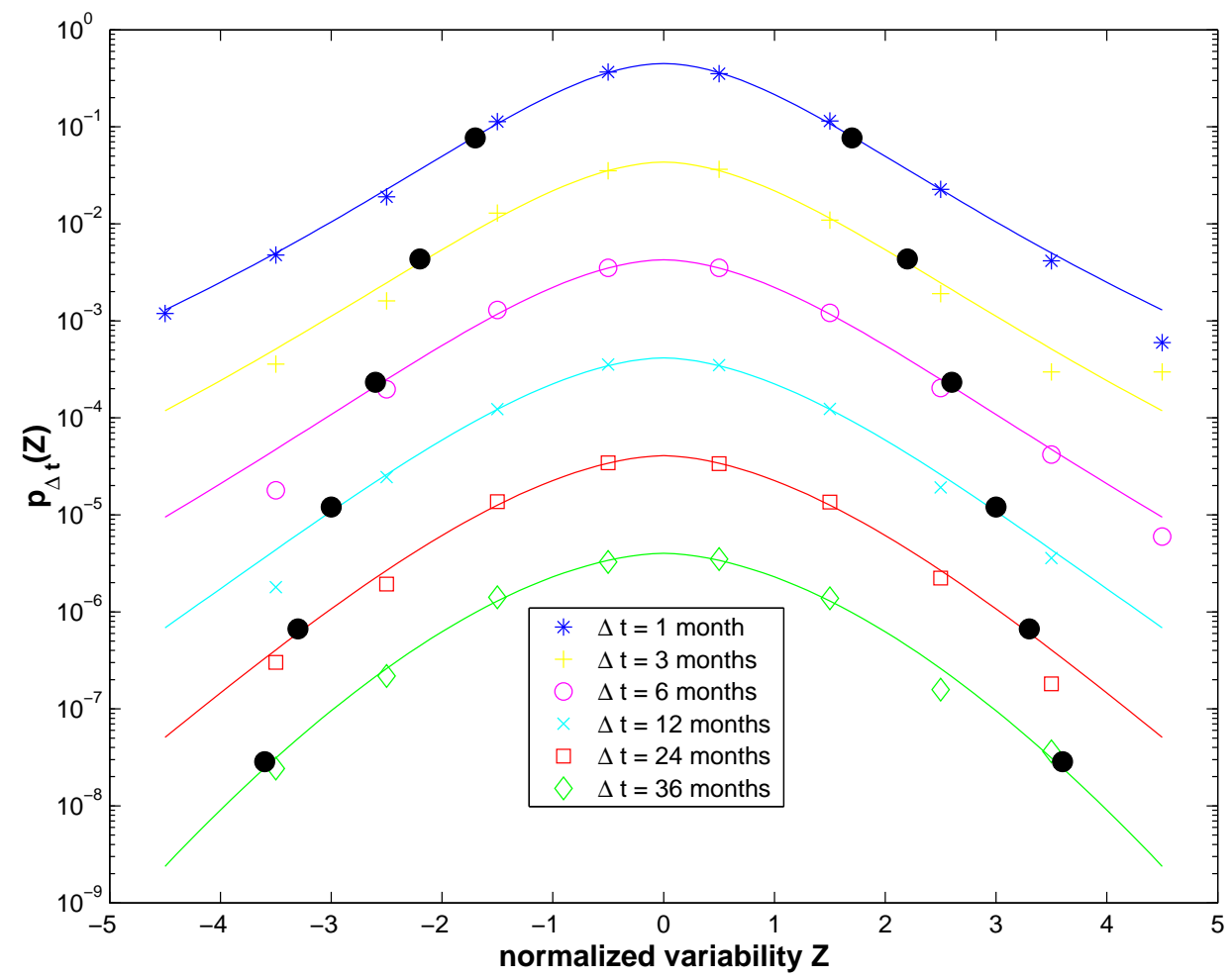

FIG. 3: Probability distribution function $(\mathrm{PDF}) p_{\Delta t}(Z)$ of normalized variability of monthly values signal of the SOI (symbols) and the Tsallis type distribution function (lines) for different values of $\Delta t=1,3,6,12,24,36$ months. The PDF (symbols and curves) for each $\Delta t$ are moved down by 10 with respect to the previous one; the curve for $\Delta t=1$ month is unmoved. The large dots mark the ends of the interval in which the distribution is like a gaussian distribution. The values of the parameters for the Tsallis type distribution function for each $\Delta t$ are summarized in Table II 


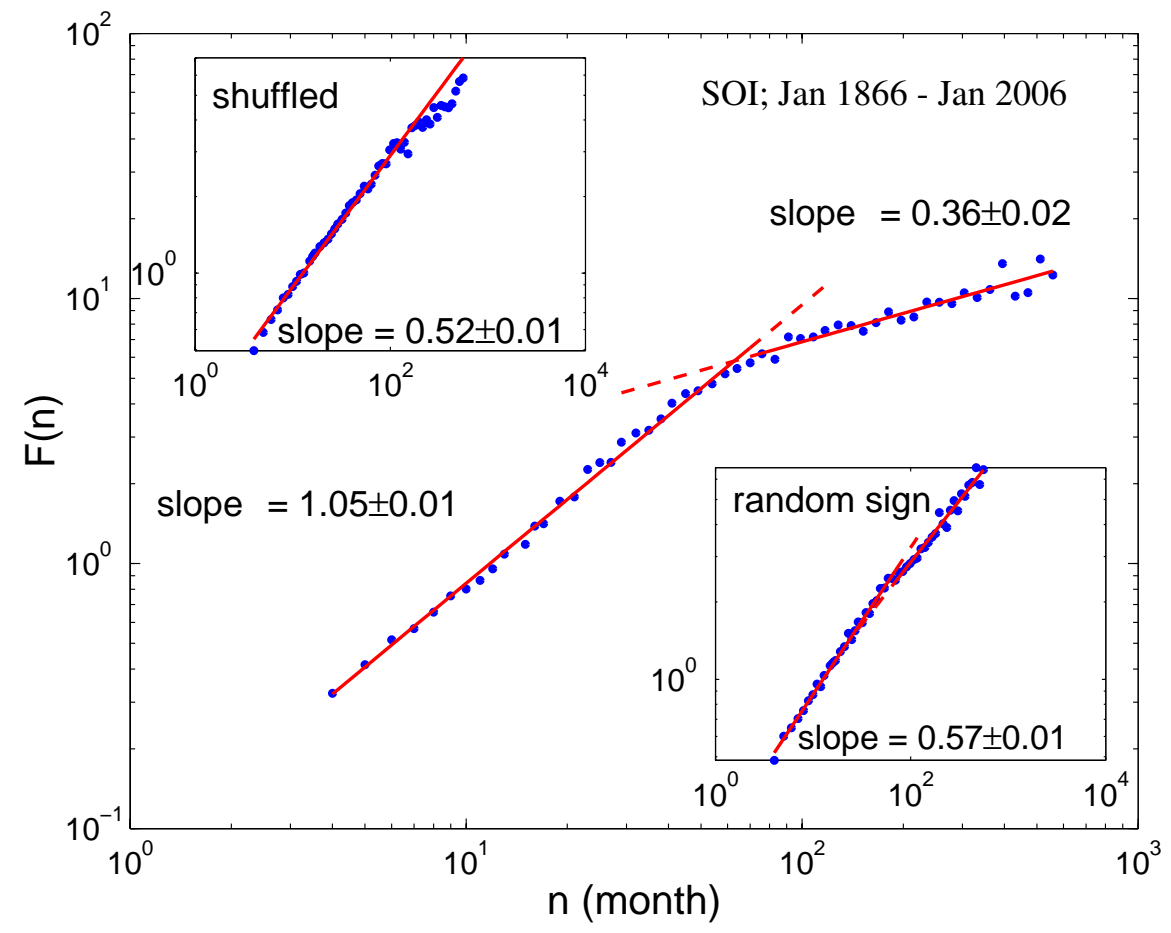

FIG. 4: DFA function $F(n)$ plotted as a function of the the box size $n$ of the integrated monthly values signal of the SOI from Jan. 1866 to Jan. 2006. $1 / f$-like fluctuations with slope $=1.06 \pm 0.01$ are obtained for time scales below 66 months and fractional Gaussian noise like fluctuations slope $=$ $0.36 \pm 0.02$ above 72 months. Insets: White noise like fluctuations of two types of surrogate data, when the data are shuffled randomly and when the sign of the data is shuffled randomly 


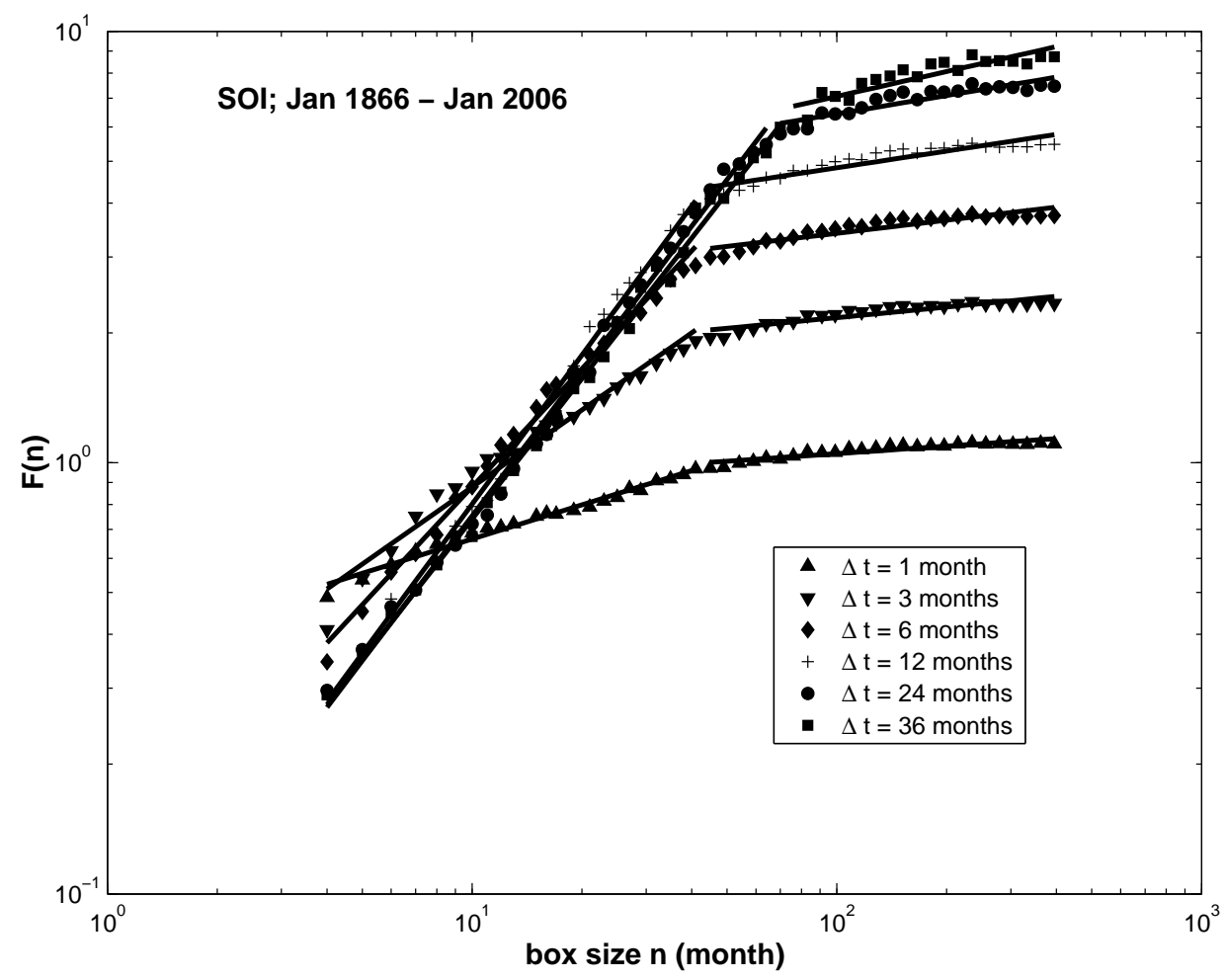

FIG. 5: DFA function $F(n)$ plotted as a function of the box size $n$ of the integrated normalized variability $Z(t, \Delta t)$ of the monthly values signal of the SOI from Jan. 1866 to Jan. 2006, for different time lags $\Delta t=1,3,6,12,24,36$ months. Values of the scaling exponents $H_{D F A}$ for the various DFA functions are summarized in Table I 


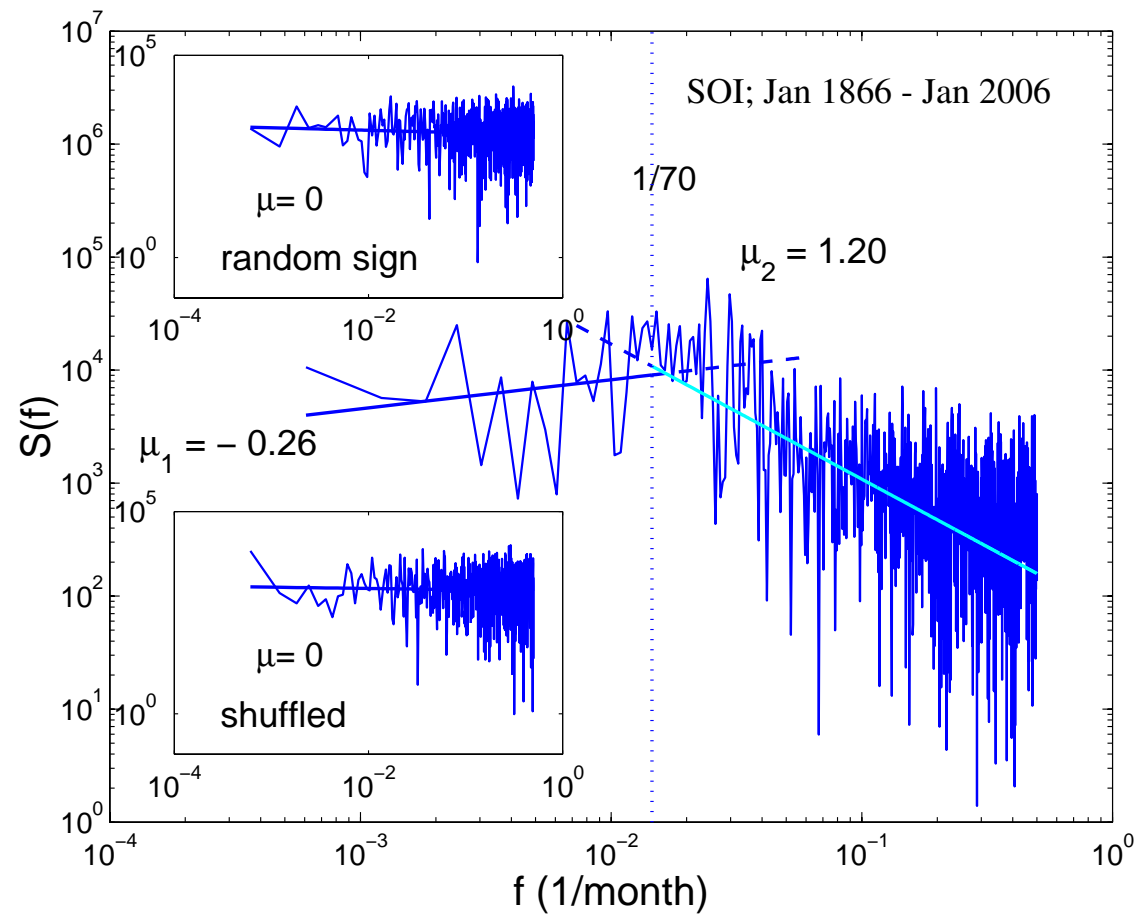

FIG. 6: Power spectrum $S(f)$ of the monthly values signal of the SOI from Jan. 1866 to Jan. 2006. A scale break at around $f=1 / 70$ month $^{-1}$ separates two scaling regions. Insets: Scaling of the power spectrum of both shuffled amplitude and shuffled sign of monthly values signal of the SOI as a white noise signal with $\mu \approx 0$ 


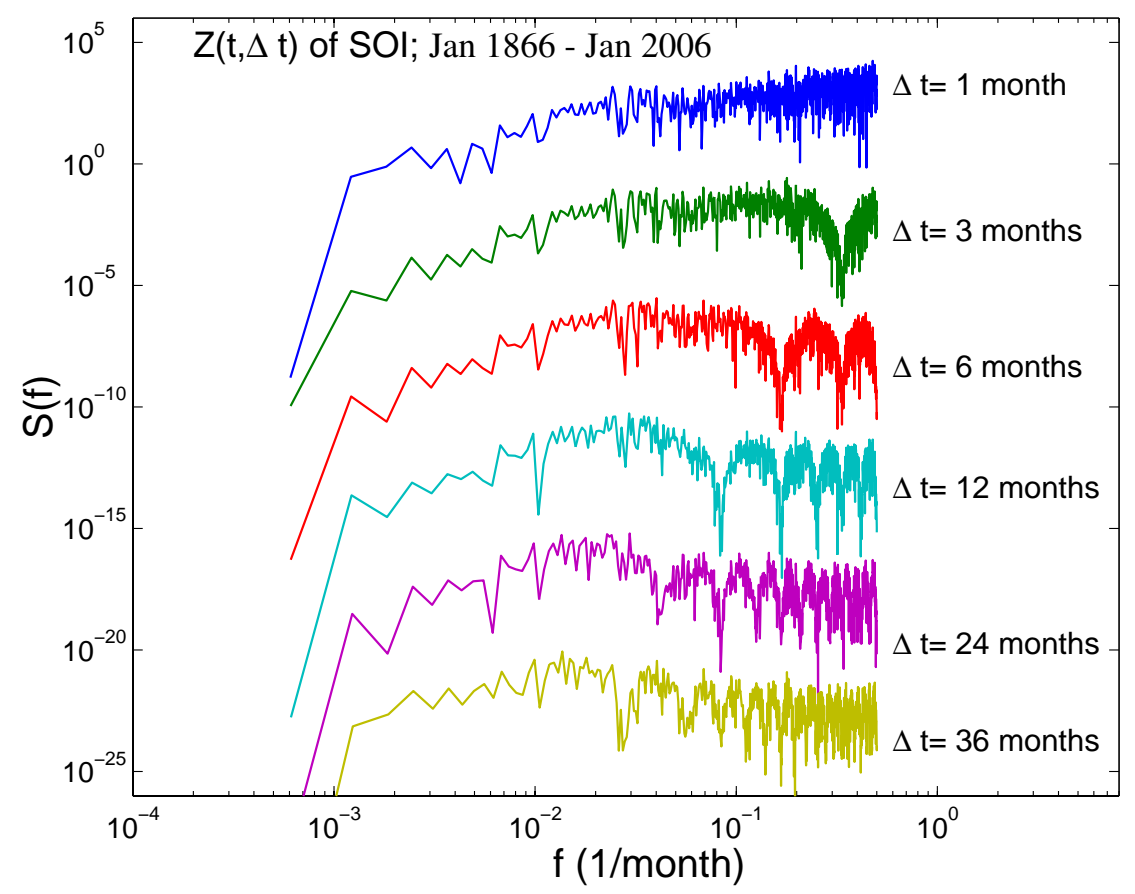

FIG. 7: Power spectrum $S(f)$ of the normalized variability $Z(t, \Delta t)$ of the monthly values signal of the SOI from Jan. 1866 to Apr. 2003 for different time lags $\Delta t=1,3,6,12,24,36$ months. Each curve is moved down by $10^{-5}$ with respect to the previous one; the power spectrum of the normalized returns for $\Delta t=1$ month is not displaced 

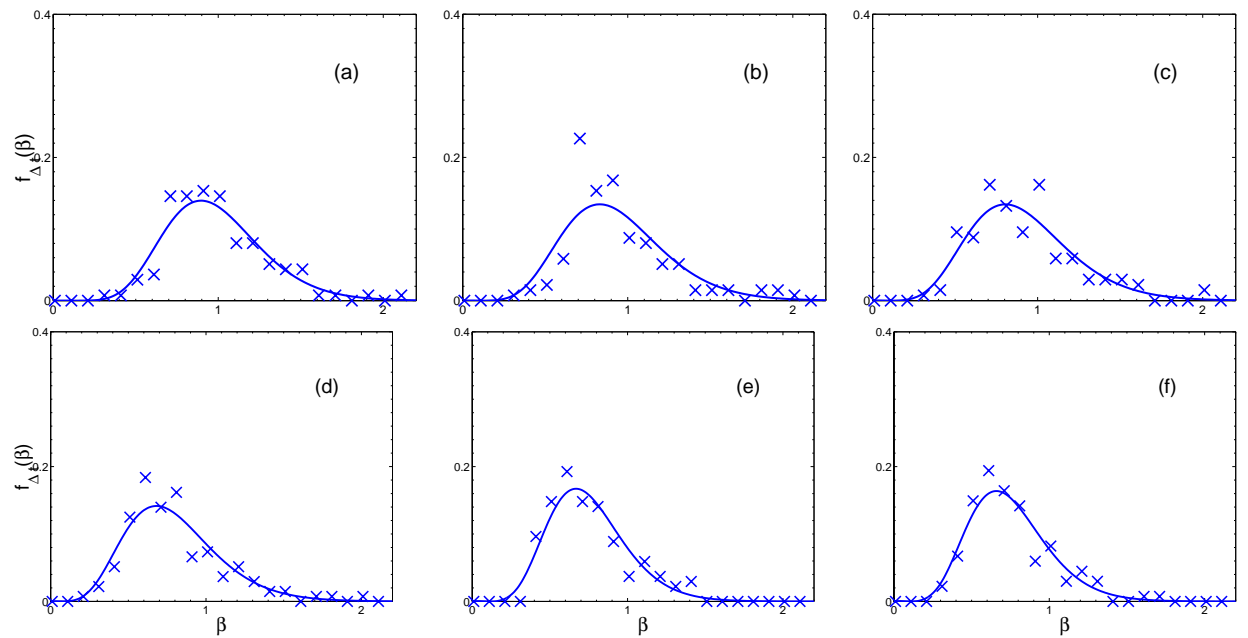

FIG. 8: Probability density $f_{\Delta t}(\beta)$ of the local volatility $\beta$ (Eq.(10)) in terms of standard deviation of the normalized variability $Z(t, \Delta t)$ of SOI in non-overlapping windows with size $m=12$ months for different time lags (symbols) (a-f) $\Delta t=1,3,6,12,24,36$ months. Lines: $\chi^{2}$-distribution as given by Eq. (7) 


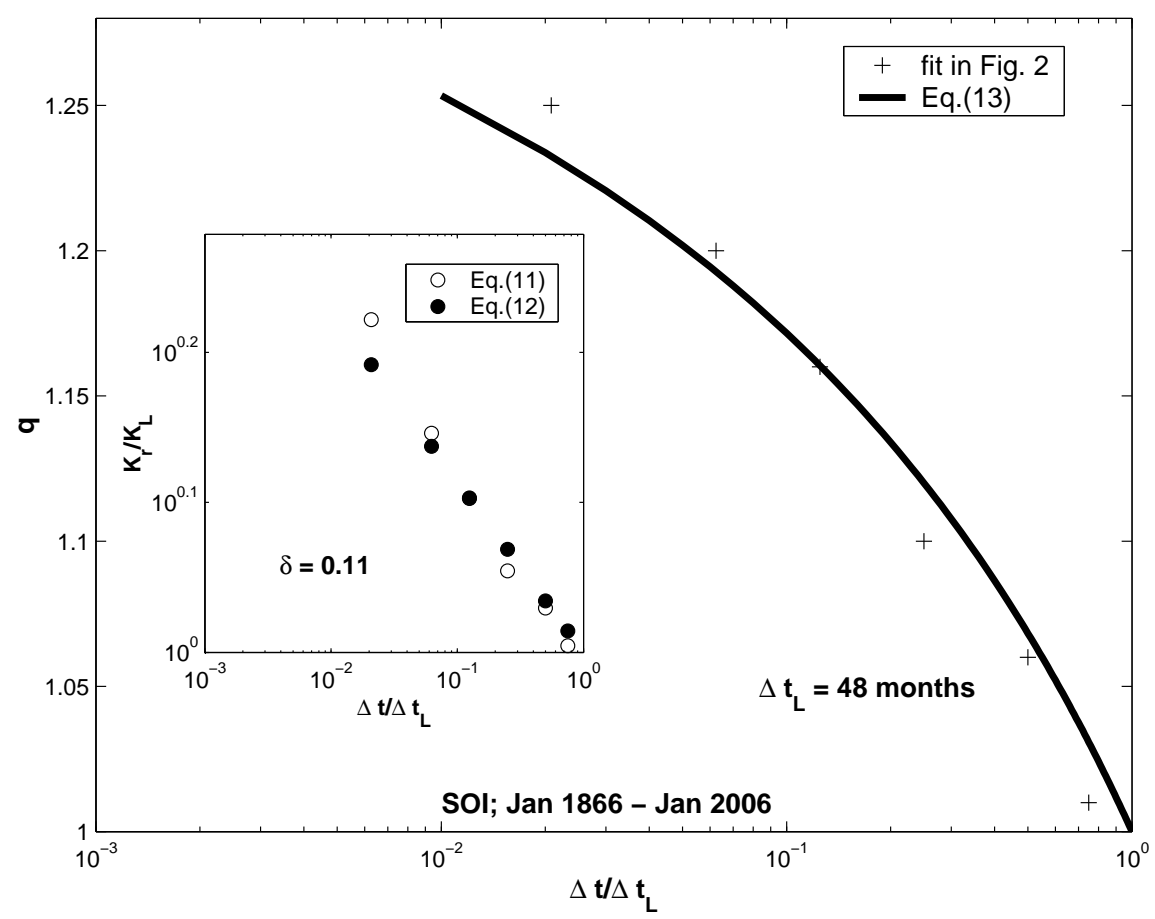

FIG. 9: The functional dependence of the Tsallis $q$ parameter on the rescaled time lag $\Delta t / \Delta t_{L}$ for $\Delta t_{L}=48$ months and $\delta=0.11$ (see Eq. (13)) (line); the symbols represent the values of the $q$ parameter listed in Table II and used to plot the fitting lines in Fig. 2. Inset : Scaling properties of the rescaled kurtosis $K_{r} / K_{L}$, where $K_{L}=3$ is the kurtosis for a Gaussian process, as a function of the rescaled time lag $\Delta t / \Delta t_{L}$ satisfying Eq. (11) (open symbols) and Eq. (12) (full symbols) 


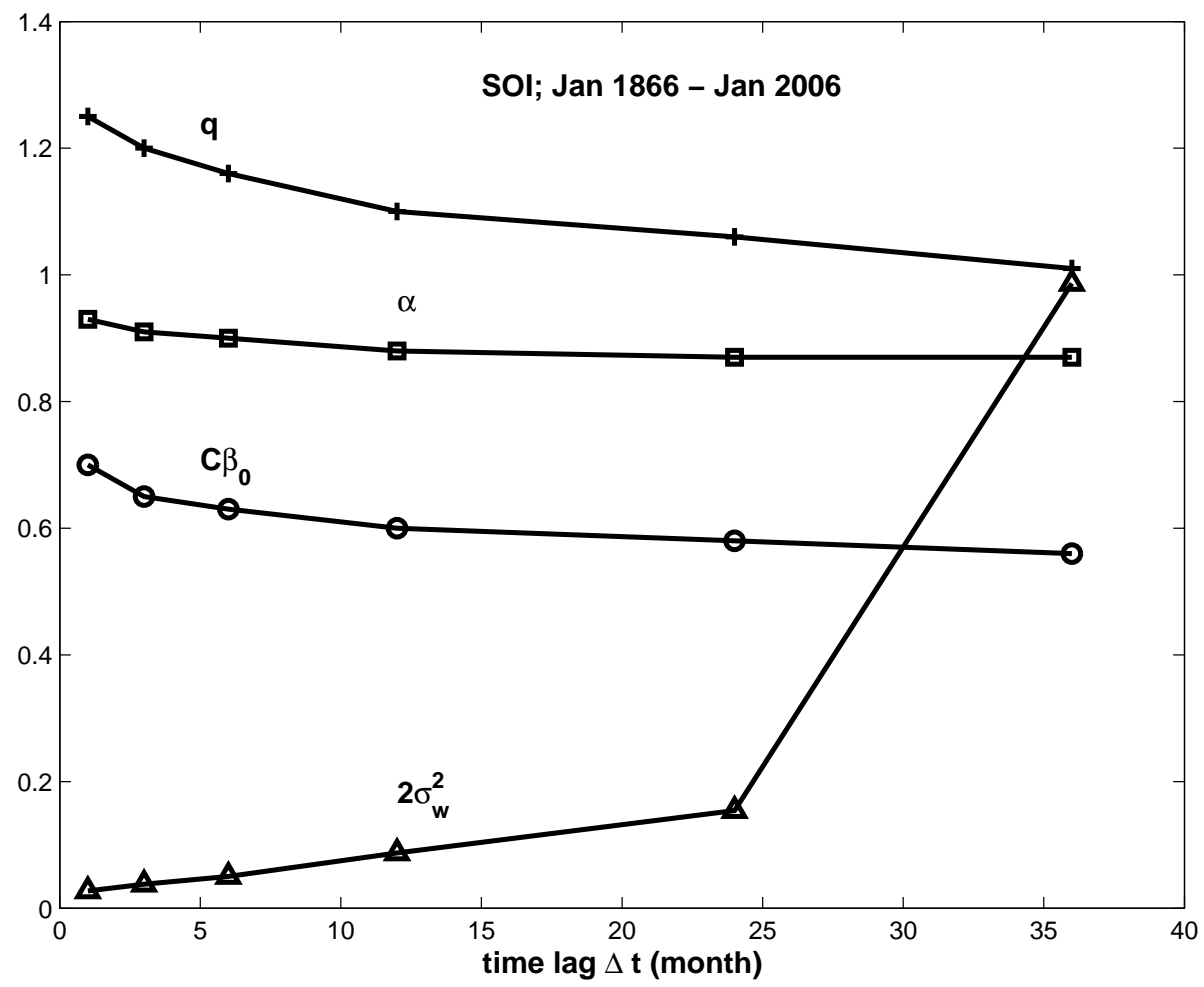

FIG. 10: Characteristic parameters of Tsallis type distribution function as defined in [33] : Tsallis $q$-parameter (crosses), $\alpha$ (squares), constant $C \beta_{0}$ used in the fit (open circles), the width of the Tsallis type distribution $2 \sigma_{w}^{2}=(2 \alpha-(q-1)) /\left(2 \alpha C \beta_{0}(q-1)\right)$ from Eq.(4) (triangles) (rescaled by a factor of $1 / 180$, for better display) 


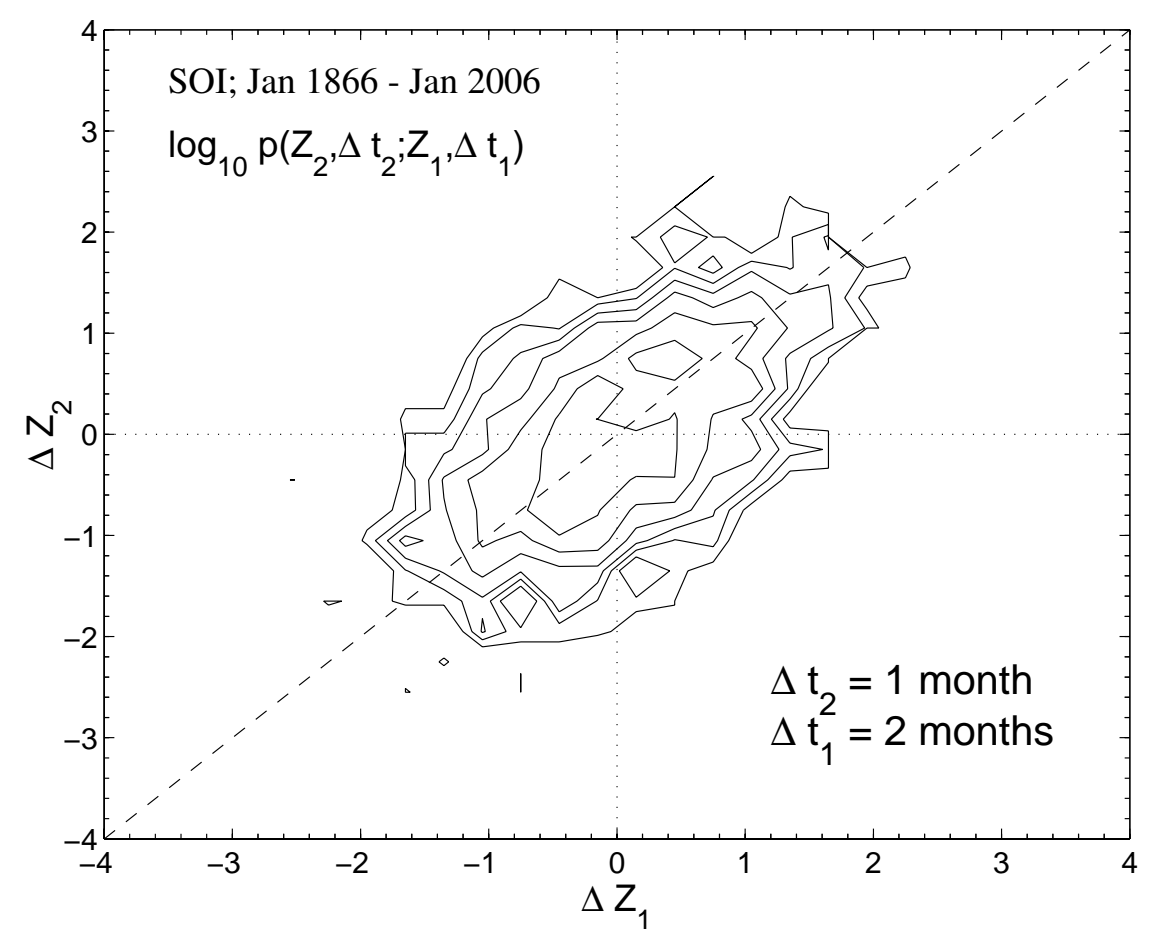

FIG. 11: Typical contour plots of the joint probability density function $p\left(Z_{2}, \Delta t_{2} ; Z_{1}, \Delta t_{1}\right)$ of the monthly values signal of the SOI from Jan. 1866 to Jan. 2006. Dashed lines have a slope +1 and emphasize the correlations between probability density functions for $\Delta t_{2}=$ 1 month and $\Delta t_{1}=2$ months. Contour levels correspond to $\log _{10} p\left(Z_{2}, \Delta t_{2} ; Z_{1}, \Delta t_{1}\right)=$ $-1.8,-2.0,-2.2,-2.4,-2.6,-2.8$ from center to border 

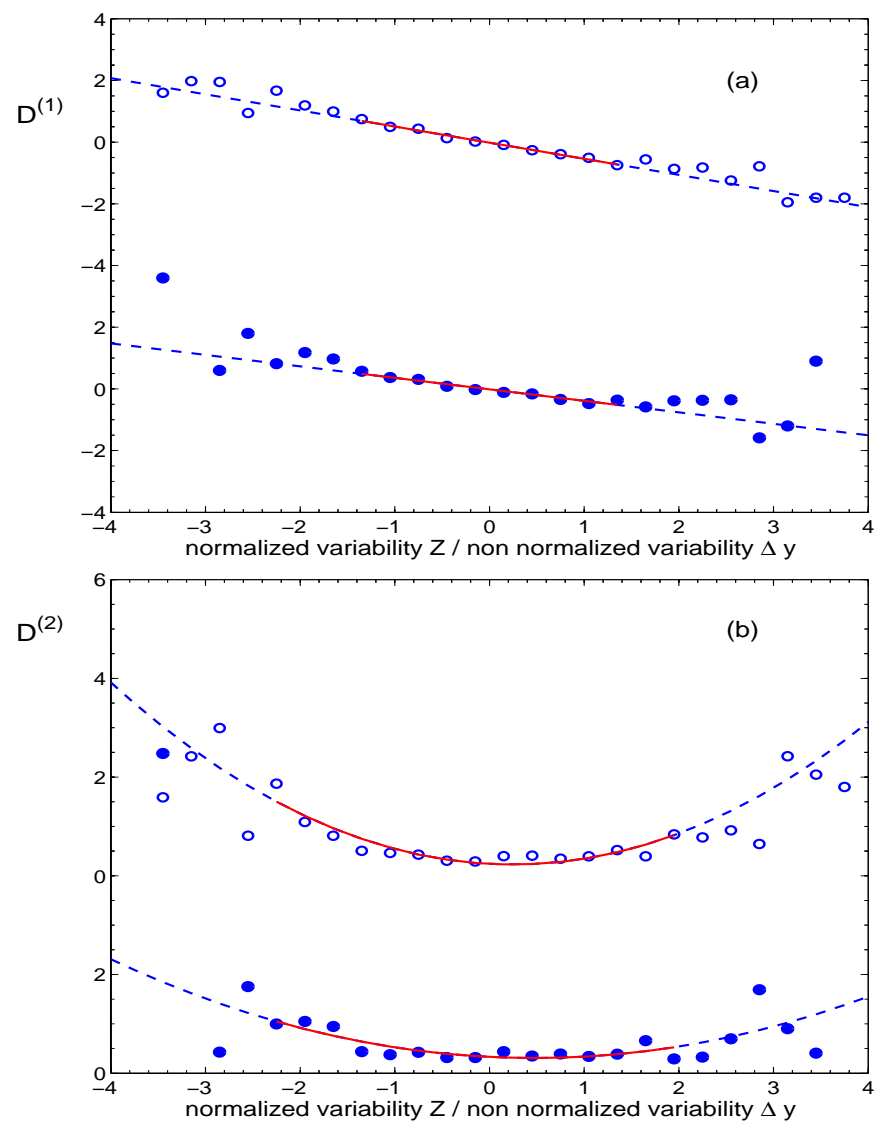

FIG. 12: Kramers-Moyal drift (a) $D^{(1)}$ and diffusion (b) $D^{(2)}$ coefficients as a function of normalized variability $Z$ (dots) and non normalized variability $Z$ (open circles) of the monthly values signal of the SOI; $D^{(1)}=-0.37 Z-0.01$ ((a) dots), $D^{(2)}=0.10 Z^{2}-0.10 Z+0.33$ ((b) dots); $D^{(1)}=$ $-0.52 \Delta y-0.02((\mathrm{a})$ open circles $), D^{(2)}=0.20 \Delta y^{2}-0.10 \Delta y+0.24$ ((b) open circles) 